www.parjournal.net

\title{
Microsurgical restoration of failed or unsatisfactory breast reconstruction: a systematic review and pooled-analysis of outcomes
}

\author{
Bassim EL-Sabawi, Anna C. Howell, Ketan M. Patel \\ Division of Plastic and Reconstructive Surgery, Keck School of Medicine of the University of Southern California, Los Angeles, CA 90033-4680, USA.
}

Correspondence to: Dr. Ketan M. Patel, Division of Plastic and Reconstructive Surgery, Keck School of Medicine of the University of Southern California, 1510 San Pablo Street, Suite 415, Los Angeles, CA 90033-4680, USA. E-mail: ketanmpatel.md@gmail.com

How to cite this article: EL-Sabawi B, Howell AC, Patel KM. Microsurgical restoration of failed or unsatisfactory breast reconstruction: a systematic review and pooled-analysis of outcomes. Plast Aesthet Res 2017;4:195-203.

\section{Article history: \\ Received: 3 Mar 2017 \\ Accepted: 21 Oct 2017 \\ Published: 31 Oct 2017 \\ Key words: \\ Autologous, \\ prosthetic, \\ salvage, \\ tertiary, \\ breast reconstruction}

\begin{abstract}
Aim: Autologous tissue transfer to salvage breast reconstruction following a previously failed or unsatisfactory reconstruction has been described by previous studies to be an effective strategy to optimize outcomes. The purpose of this systematic review is to assimilate the relevant literature to evaluate surgical and aesthetic outcomes following autologous breast reconstruction in the setting of a prior unsuccessful reconstruction. Methods: A systematic review of the English literature was performed on Pubmed/MEDLINE to identify all manuscripts reporting surgical outcomes, aesthetic outcomes, or patient satisfaction of autologous breast reconstruction in the setting of a previously failed or unsatisfactory breast reconstruction. Results: Nineteen studies met the criteria for inclusion. Of these, 15 studies reported outcomes following autologous conversion of unsuccessful prosthetic reconstruction (778 breasts). Pooled-analysis of these studies demonstrated total flap loss in $1.6 \%$, microsurgical revision in $3.2 \%$, total complications in $21.7 \%$, and revision surgery in $26.5 \%$. Review of these studies demonstrated high rates of positive aesthetic outcomes and patient satisfaction. Five studies (54 patients) evaluated outcomes following autologous salvage of prior unsuccessful autologous breast reconstruction. Pooled-analysis of these studies demonstrated no instances of microsurgical revision, total complications in $42.2 \%$, and revision surgery in $70.0 \%$. Total flap failure following tertiary reconstruction utilizing microsurgical free flaps occurred in $9.5 \%$. Data describing aesthetic outcomes or patient satisfaction was lacking in these studies. Conclusion: Autologous conversion in the setting of unsuccessful prosthetic breast reconstruction appears to be valuable option to improve outcomes. There is little evidence to suggest that prior prosthetic reconstruction negatively impacts outcomes of autologous breast reconstruction. Data describing autologous breast reconstruction following prior flap loss is limited but suggests it is viable method to salvage breast reconstruction in appropriate patients.
\end{abstract}

This is an open access article licensed under the terms of Creative Commons Attribution 4.0 International License (https://creativecommons.org/licenses/by/4.0/), which permits unrestricted use, distribution, and reproduction in any medium, as long as the original author is credited and the new creations are licensed under the identical terms.

For reprints contact: service@oaepublish.com

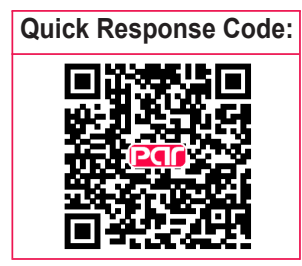




\section{INTRODUCTION}

Breast reconstruction has consistently been shown to enhance body image and quality of life in breast cancer patients undergoing mastectomy ${ }^{[1-4]}$. Despite this, patients suffering reconstructive failures or unsatisfactory outcomes are not negligible ${ }^{[5,6]}$. Previously failed breast reconstruction provides multifaceted and unique challenges for plastic surgeons. Following evaluation of the causes of failure and reassessment of patient goals, autologous tissue transfer to salvage breast reconstruction has been shown to be an effective strategy to optimize outcomes ${ }^{[6-8]}$. Also termed tertiary reconstruction by previous studies, autologous salvage has been reported as a means of breast restoration following both failed prosthetic breast reconstruction and previously failed autologous reconstruction ${ }^{[7,8]}$.

The majority of the literature pertaining to tertiary reconstruction describes autologous conversion following complications with prosthetic reconstruction ${ }^{[7,9-11]}$. Prosthetic reconstruction is the most common method of breast reconstruction and has increased at a rate of $5 \%$ a year ${ }^{[12]}$. Despite its increasing popularity, complications leading to poor aesthetics, persistent pain, and implant loss are not uncommon, particularly in the setting of adjuvant radiotherapy ${ }^{[13,14]}$. In addition to alleviating implant-related complications, autologous conversion may provide a more natural appearance and improve longevity of the reconstructed breast. However, the impact of a prior complicated prosthetic reconstruction on outcomes of autologous conversion remains unclear. A recent study has suggested that patients with unsatisfactory prior prosthetic reconstruction have increased recipient vessel scarring and major complications with autologous conversion as compared to patients that undergo de novo autologous reconstruction ${ }^{[10]}$.

Tertiary reconstruction following failed autologous reconstruction by utilization of a second flap has also been described ${ }^{[8]}$. Although uncommon, flap failure in autologous breast reconstruction is a stressful and demanding situation for the patient and the surgeon ${ }^{[15]}$. Use of an additional flap to salvage breast reconstruction may provide a means to still obtain an acceptable reconstructive result and mitigate patient distress associated with flap failure. Despite demonstrating success in previous reports, questions concerning the risk of reattempting autologous reconstruction with a different flap exist as risk factors that contributed to the initial flap failure and sequelae of the previous surgery may compromise its survival ${ }^{[8,16]}$. In addition, data evaluating aesthetic outcome and quality of life following tertiary reconstruction of failed autologous breast reconstruction are lacking and further add to the confusion regarding its potential benefit.

Due to the infrequency of failed or unsatisfactory breast reconstruction, management of this group of patients in the literature is primarily limited to several small case series from a few centers ${ }^{[7,8,16]}$. As a result, the indications, methods, and expected outcomes of tertiary reconstruction are poorly defined. The purpose of this systematic review is to assimilate the relevant literature to evaluate surgical and aesthetic outcomes following tertiary reconstruction. The goal is to provide surgeons with a foundation of knowledge to assess the risks and benefits associated with autologous salvage of a previously unsuccessful breast reconstruction.

\section{METHODS}

A systematic search of the literature published from January 1, 1980 to December 29, 2016 was performed using search terms "salvage", "tertiary", "restoration" and "breast reconstruction" to identify all relevant articles on Pubmed/MEDLINE. Inclusion criteria included studies that reported surgical outcomes, aesthetic outcomes, or patient satisfaction of autologous breast reconstruction following failed or unsatisfactory breast reconstruction. The reference lists of all included studies were reviewed to identify relevant articles that may have not been captured in the search. Exclusion criteria included studies that reported relevant outcomes of less than 5 patients, non-English language articles, reviews, and studies reporting previously published data. Studies reporting management of patients with complicated or failed reconstruction that did not adequately describe outcomes of the autologous salvage procedure were excluded. One reviewer performed the search protocol and article selection (B.E.), and two reviewers (K.M.P. and A.C.H.) reconciled any discrepancies.

Adherence to the standardized methodologic principles of Preferred Reporting Items for Systematic Reviews and Meta-Analyses for reporting of systematic reviews guided the analysis ${ }^{[17]}$. Study characteristics, patient demographics and comorbidities, oncologic treatment characteristics, and details regarding the initial failed breast reconstruction were extracted. Primary outcomes included flap choice for autologous salvage, total complications, microsurgical revisions, total flap loss, and revision procedures associated with tertiary reconstruction. Secondary outcomes of tertiary reconstruction included hematoma, seroma, infection, fat necrosis, partial flap loss/skin necrosis, and measures of aesthetic outcome or patient satisfaction. 


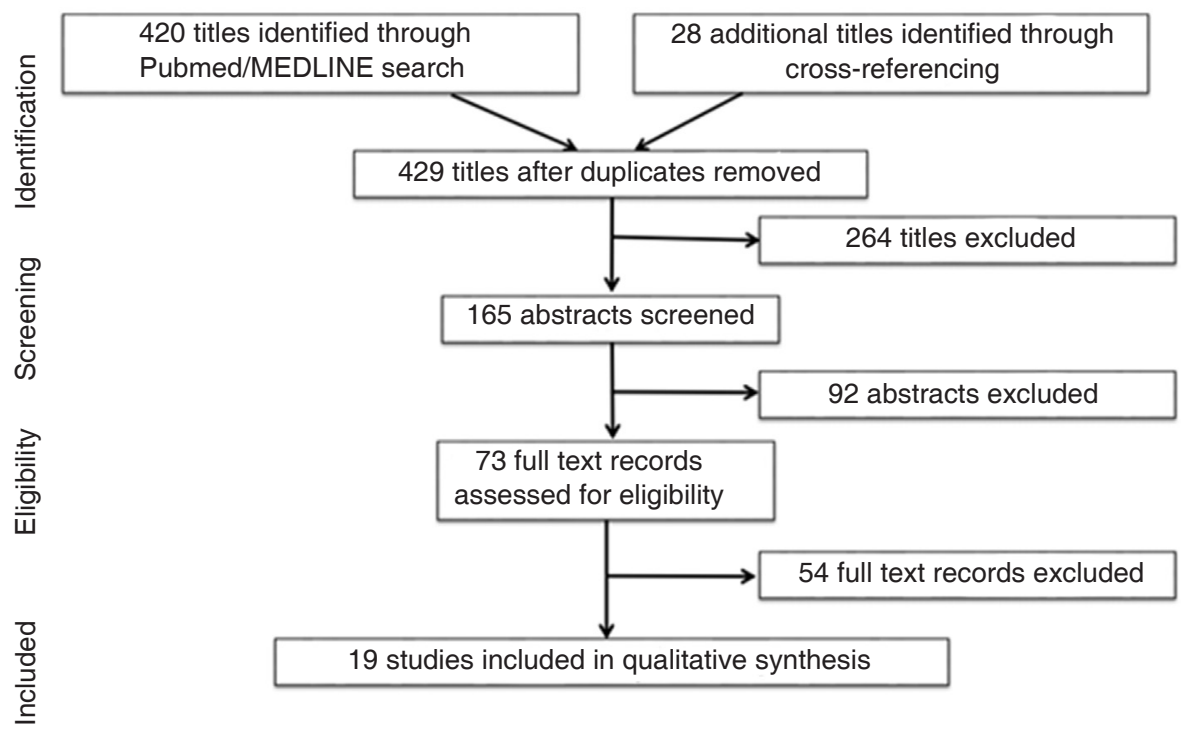

Figure 1: Flowchart of article selection process

Data analysis was completed using simple means allocating weight to each study by sample size. If only the median was reported, then a Gaussian distribution was assumed and the medians were equated to means. Outcomes were stratified by method of failed/ unsatisfactory initial breast reconstruction. Qualitative analysis of aesthetic outcome/patient satisfaction is described due to the heterogeneity of included studies. Statistical significance was defined as $P<0.05$.

\section{RESULTS}

Results from the initial search yielded 420 titles. Screening yielded 165 studies for abstract review, of which 73 were selected for full text review. Ultimately, 19 studies met inclusion criteria and were included in this review [Figure 1]. There were no randomized clinical trials, 17 studies (89.5\%) were retrospective, and 2 studies $(10.5 \%)$ were prospective. Study characteristics and patient demographics and comorbidities within included studies are described in Table 1.

\section{Autologous salvage of unsuccessful prosthetic breast reconstruction}

Fifteen studies evaluated outcomes following autologous conversion of failed or unsatisfactory prosthetic reconstruction including a total of 564 patients (778 breasts) $)^{[6,7,9-11,18-27]}$. Study size weighted mean age was 49.3 years and mean body mass index (BMI) was $26.6 \mathrm{~kg} / \mathrm{m}^{2}$. Mean follow-up was 23.5 months. Thirteen of these studies (719 breasts) reported implant related complications occurring prior to autologous conversion ${ }^{[6,7,9-11,18,19,21-24,26,27]}$. Capsular contracture was the most commonly cited implant related complication with modified Baker grade IIIIV contracture occurring in 168 breasts (23.4\%), modified Baker grade I-II contracture in 137 breasts $(19.1 \%)$, and uncharacterized capsular contracture reported in 144 breasts $(20.0 \%)$. Other commonly reported indications for autologous conversion were poor cosmesis or asymmetry in 102 breasts (14.1\%), pain/discomfort in 72 breasts $(10.0 \%)$, infection/ exposure in 71 breasts $(9.9 \%)$, implant rupture in 25 breasts $(3.5 \%)$, or recurrence in 12 breasts $(1.6 \%)$. Neoadjuvant or adjuvant radiotherapy prior to autologous conversion was reported in 231 patients $(48.8 \%)$. The deep inferior epigastric perforator (DIEP) flap was the most commonly utilized flap for autologous conversion and was reported in 398 breasts $(51.1 \%)$. Other common flaps choices included a free transverse abdominis myocutaneous (fTRAM) flap in 89 breasts $(11.4 \%)$, superior gluteal artery perforator (SGAP) flap in 67 breasts $(8.6 \%)$, transverse upper gracilis flap or transverse myocutanous gracilis (TMG) flap in 65 breasts $(8.4 \%)$, superficial inferior epigastric artery (SIEA) flap in 53 breasts $(6.8 \%)$, inferior gluteal artery perforator flap in 35 breasts (4.5\%), latissimus dorsi (LD) flap with or without implant in 27 breasts $(3.5 \%)$, or other or undescribed flaps in the remaining 29 breasts $(3.7 \%)$.

Thirteen studies ( $n=748$ flaps) reported complications following autologous salvage of complicated prosthetic reconstruction [Table 2] $]^{[6,7,9-11,18,20-24,26,27]}$. A total of 12 total flap losses (1.6\%; range $0-6.9 \%)$ were reported, including 3 DIEP flap loss, 3 SGAP flap loss, 2 fTRAM flap loss, and flap loss in 4 breasts that were not described. Microsurgical revisions were reported in 20 
Table 1: Study characteristics and patient demographics of included studies

\begin{tabular}{|c|c|c|c|c|c|c|c|c|c|}
\hline Authors & Study type & $\begin{array}{l}\text { Patients } \\
\text { (n) }\end{array}$ & $\begin{array}{c}\text { Years of } \\
\text { patient } \\
\text { inclusion }\end{array}$ & $\begin{array}{c}\text { Mean } \\
\text { age } \\
\text { (year) }\end{array}$ & $\begin{array}{c}\text { Mean } \\
\text { BMI } \\
\left(\mathrm{kg} / \mathrm{m}^{2}\right)\end{array}$ & $\begin{array}{c}\text { Smoking } \\
(\%)\end{array}$ & $\begin{array}{c}\text { Prior } \\
\text { chemotherapy } \\
(\%)\end{array}$ & $\begin{array}{c}\text { Prior } \\
\text { y radiation } \\
(\%)\end{array}$ & $\begin{array}{l}\text { Follow- } \\
\text { up } \\
\text { (month) }\end{array}$ \\
\hline Munhoz et al. ${ }^{[16]} 2016$ & Retrospective & 12 & $1999-2013$ & 47.3 & - & - & 66.6 & 66.6 & 42.5 \\
\hline Roostaeian et al. ${ }^{[10]} 2016$ & Retrospective & 89 & $2005-2014$ & 51.3 & 26.5 & 5 & - & 48.7 & 10.2 \\
\hline Pülzl et al..$^{[18]} 2015$ & Retrospective & 33 & $2006-2011$ & $46^{*}$ & - & - & - & - & 51.6 \\
\hline Mioton et al..$^{[19]} 2014$ & Retrospective & 18 & $2004-2010$ & 50.5 & 29.4 & 0 & 58.8 & 41.4 & - \\
\hline Mohan et al. ${ }^{[20]} 2013$ & Retrospective & 29 & $2004-2010$ & 50.5 & 26.1 & 16.1 & - & 52.3 & 20 \\
\hline Rabey et al. ${ }^{[21]} 2013$ & Retrospective & 14 & 2000-2012 & $50^{*}$ & - & 7.1 & 50.0 & 85.7 & 21 \\
\hline Spear et al. ${ }^{[6]} 2013$ & Retrospective & 7 & 2005-2010 & - & 28.5 & - & - & 43.3 & - \\
\hline Peled et al. ${ }^{[22]} 2012$ & Prospective & 5 & $2005-2007$ & 45.4 & 25.9 & 3.5 & 52.0 & 51.8 & 52.5 \\
\hline Levine et al. ${ }^{[9]} 2011$ & Prospective & 191 & $1998-2008$ & $49^{*}$ & - & - & - & 20.0 & - \\
\hline Hamdi et al. ${ }^{[7]} 2010$ & Retrospective & 54 & 2002-2009 & 46.8 & - & 7.4 & 72.2 & 74.0 & 31 \\
\hline Hamdi et al. ${ }^{[8]} 2010$ & Retrospective & 8 & $2002-2009$ & 46.7 & 24 & 21.4 & 86.0 & 35.7 & 37 \\
\hline Visser et al. ${ }^{[11]} 2010$ & Retrospective & 42 & $2001-2007$ & $53^{*}$ & $26^{*}$ & - & - & 27.9 & $24^{*}$ \\
\hline Hammond et al. ${ }^{[28]} 2007$ & Retrospective & 14 & 1992-2002 & 48 & - & 14.3 & - & 35.7 & - \\
\hline Gurunluoglu et al. ${ }^{[23]} 2005$ & Retrospective & 7 & $1994-2001$ & 45.7 & - & - & - & - & 57.6 \\
\hline Mosahebi et al. ${ }^{[24]} 2005$ & Retrospective & 5 & - & 55 & - & - & - & 0 & 15 \\
\hline Karanas et al. ${ }^{[29]} 2002$ & Retrospective & 7 & - & 54 & - & - & - & 14.3 & - \\
\hline Spear and Onyewu ${ }^{[25]} 1999$ & Retrospective & 19 & 1990-1997 & - & - & - & - & 100 & - \\
\hline Weiss and Ship ${ }^{[26]} 1995$ & Retrospective & 26 & - & 47.4 & - & - & - & - & \\
\hline Feng et al. ${ }^{[27]} 1994$ & Retrospective & 33 & 1988-1993 & 47 & - & 33 & - & - & - \\
\hline
\end{tabular}

${ }^{*}$ median. BMI: body mass index

flaps $(3.2 \%$; range $0-3.7 \%)$. Complications requiring surgery were reported in $10.0 \%$ (range $0-17.4 \%$ ) of flaps and total complications were reported in $21.7 \%$ (range 10.0-34.4\%) of flaps. Of these complications, hematoma was reported in 18 flaps (2.7\%; range $0-7.7 \%)$, seroma in 9 flaps $(1.9 \%$; range $0-2.5 \%)$, infection in 10 flaps (3.5\%; range $0-11.1 \%)$, wound healing problems in 9 flaps $(3.7 \%$; range $0-7.1 \%)$, and fat necrosis 18 in flaps (3.5\%; range $0-4.8 \%$ ). Breast related revisional surgery to improve aesthetic outcome was reported in 84 patients $(26.5 \%$; range $4.6-80 \%)$. A single study compared outcomes of patients with free flap breast reconstruction following complicated prosthetic reconstruction to those with de novo autologous reconstruction $(n=178)^{[10]}$. No difference was observed in flap loss $(2.5 \%$ vs. $2.4 \%$, $P=1.00)$ or total complications $(27.2 \%$ vs. $26.0 \%, P=$ 0.89 ) between the two cohorts.

Measures of aesthetic outcome or patient satisfaction were reported by 5 studies including 147 patients ${ }^{[11,19,20,25,27]}$. Review of these studies demonstrated significant heterogeneity in methods of evaluation, rating scales, and reporting of aesthetic outcomes. Aesthetic means based on numerical rating scales were reported in 3 studies ${ }^{[11,19,25]}$. Utilizing a 5-point Likert scale (5, very satisfied; 1 , very dissatisfied), one study reported numerical means of self-reported assessments in 29 patients with scores of 4.24 for breast volume, 4.16 for breast shape, 3.83 for symmetry, 3.92 for breast scars, and 3.42 for nipple/
NAC complex ${ }^{[11]}$. Another study utilized a 4-point scale (4, excellent; 1 , poor) to evaluate 14 irradiated implant reconstructions with later addition of a TRAM or LD flap $^{[25]}$. They reported a mean overall aesthetic score of 3.25 , which was similar to the mean score of 3.28 in patients with non-irradiated implant reconstruction. A validated 3-point scale (0-2) of 5 distinct aesthetic domains was used by another study, which reported mean scores of 1.6 for volume, 1.6 for contour, 1.75 for placement, 1.80 for inframammary fold, and 1.35 for scarring in 18 patients who had autologous conversion after experiencing complication with initial expanderimplant reconstruction ${ }^{[19]}$. They reported superior scores across 4 of these domains (volume, contour, placement, and inframammarry fold) as compared to patients completed expander implant reconstruction without complication. Two of the studies (71 patients) reported proportions of patients satisfied with the aesthetic result following tertiary reconstruction, with satisfaction rates ranging $84-89 \%{ }^{[11,20]}$. Lastly, one study noted that $92 \%$ of their sample of 25 patients reported improved cosmesis with autologous conversion than with prior implant reconstruction ${ }^{[27]}$.

\section{Autologous salvage of prior unsuccessful autologous breast reconstruction}

Five studies (54 patients) evaluated outcomes following autologous salvage of prior unsuccessful autologous breast reconstruction [Table 3$]^{[8,16,20,28,29]}$. All of these studies were small retrospective case series. Study size weighted mean age was 48.6 and mean 
Table 2: Studies reporting surgical outcomes of autologous conversion following failed or unsatisfactory prosthetic-based reconstruction

\begin{tabular}{|c|c|c|c|c|c|c|c|}
\hline \multirow[b]{2}{*}{ Authors } & \multirow[b]{2}{*}{$\begin{array}{l}\text { Breasts } \\
(n)\end{array}$} & \multirow[b]{2}{*}{$\begin{array}{l}\text { Indications for } \\
\text { autologous } \\
\text { conversion }\end{array}$} & \multirow[b]{2}{*}{ Flap choice } & \multicolumn{4}{|c|}{ Surgical outcomes } \\
\hline & & & & $\begin{array}{l}\text { Microsurgical } \\
\text { revisions, } \\
n(\%)\end{array}$ & $\begin{array}{l}\text { Total } \\
\text { flap } \\
\text { loss, } \\
n(\%)\end{array}$ & $\begin{array}{c}\text { Total } \\
\text { complications } \\
n(\%)\end{array}$ & $\begin{array}{c}\text { Revisional- } \\
\text { surgery", } \\
n(\%)\end{array}$ \\
\hline Roostaeian et al. ${ }^{[10]} 2016$ & 121 & $\begin{array}{l}62 \% \text { CC; } 13 \% \text { rupture; } \\
7 \% \text { mastectomy; infection }\end{array}$ & $\begin{array}{l}60 \% \text { DIEP; } 26 \% \\
\text { fTRAM; SIEA 7\%; }\end{array}$ & $2(1.7)$ & $4(2.5)$ & $33(27.2)$ & - \\
\hline Pülzl et al. ${ }^{[18]} 2015$ & 52 & $\begin{array}{c}4 \% ; 13 \% \text { other } \\
61 \% \text { Grade I-II CC; } \\
39 \% \text { grade III-IV CC; } \\
85 \% \text { pain; } 73 \% \text { foreign } \\
\text { body sensation; } 52 \% \\
\text { asymmetry }\end{array}$ & $\begin{array}{c}\text { SGAP 5\%; Other } 2 \% \\
\text { Depithelialized free } \\
\text { TMG }\end{array}$ & 0 & 0 & - & $13(81.3)^{\mathrm{C}}$ \\
\hline Mohan et al. ${ }^{[20]} 2013$ & 29 & $\begin{array}{l}\text { Grade III-IV CC, } \\
\text { asymmetry, extrusion, } \\
\text { exposure, or poor } \\
\text { cosmesis }\end{array}$ & $\begin{array}{l}\text { 76\% DIEP; } 14 \% \text { LD } \\
\text { +/- implant; } 10 \% \\
\text { fTRAM }\end{array}$ & - & $2(6.9)$ & - & - \\
\hline Rabey et al. ${ }^{[21]} 2013$ & 14 & $\begin{array}{c}100 \% \text { poor cosmesis; } \\
86 \% \text { pain; } 64 \% \text { CC; } 43 \% \\
\text { tightness }\end{array}$ & $\begin{array}{c}\text { 64\% DIEP; } 36 \% \\
\text { msTRAM }\end{array}$ & - & 0 & $2(14.2)$ & $1(7.1)$ \\
\hline Spear et al. ${ }^{[6]} 2013$ & 7 & $\begin{array}{l}66 \% \text { infection; } 24 \% \\
\text { exposure; } 10 \% \text { infection } \\
\text { and exposure }\end{array}$ & $\begin{array}{l}\text { 43\% DIEP; } 29 \% \\
\text { LD + implant; } 14 \% \\
\text { msTRAM; } 14 \% \text { LD }\end{array}$ & - & 0 & - & - \\
\hline Peled et al. ${ }^{[22]} 2012$ & 5 & $100 \%$ infection & DIEP or TRAM & - & 0 & - & - \\
\hline Levine et al. ${ }^{[9]} 2011$ & 284 & $\begin{array}{l}46 \% \text { grade III-IV CC; } \\
41 \% \text { grade I-II CC; } 8 \% \\
\text { infection; } 5 \% \text { other }\end{array}$ & $\begin{array}{c}58 \% \text { DIEP; } 18 \% \\
\text { SGAP; } 12 \% \text { IGAP; } \\
\text { 11\% SIEA; } 2 \% \text { TUG }\end{array}$ & $11(3.7)$ & $3(1.1)$ & $57(20.1)$ & $13(4.6)$ \\
\hline Hamdi et al. ${ }^{[7]} 2010$ & 81 & $\begin{array}{c}46 \% \text { poor cosmesis; } 24 \% \\
\text { infection/exposure; } 24 \% \\
\text { CC; } 4 \% \text { recurrence }\end{array}$ & $\begin{array}{l}81 \% \text { DIEP; } 14 \% \\
\text { SIEA; } 5 \% \text { TMG }\end{array}$ & $4(4.9)$ & $1(1.2)$ & - & $29(53)$ \\
\hline Visser et al. ${ }^{[11]} 2010$ & 61 & $\begin{array}{l}68 \% \text { pain/tightness; } 64 \% \\
\text { poor cosmesis; } 14 \% \\
\text { infection }\end{array}$ & $\begin{array}{c}\text { 77\% DIEP; } 16 \% \\
\text { mini-TRAM; } 7 \% \\
\text { TMG }\end{array}$ & $2(3.3)$ & 0 & $21(34.4)$ & $19(45)$ \\
\hline Gurunlougu et al. ${ }^{[23]} 2005$ & 14 & $100 \%$ grade III-IV CC & $\begin{array}{l}\text { 43\% msTRAM; } 36 \% \\
\text { DIEP; } 21 \% \text { SIEA }\end{array}$ & - & 0 & $1(7.1)$ & - \\
\hline Mosahebi et al. ${ }^{[24]} 2005$ & 10 & $\begin{array}{l}100 \% \text { grade III-IV CC, } \\
\text { pain; and poor cosmesis }\end{array}$ & DIEP & 0 & 0 & $1(10.0)$ & - \\
\hline Weiss and Ship ${ }^{[26]} 1995$ & 18 & $\begin{array}{l}89 \% \text { CC; } 22 \% \text { implant } \\
\text { failure; } 11 \% \text { pain }\end{array}$ & $\begin{array}{l}61 \% \text { DMP; } 39 \% \\
\text { PTRAM }\end{array}$ & - & 0 & $4(22.2)$ & - \\
\hline Feng et al. ${ }^{[27]} 1994$ & 52 & $\begin{array}{c}82 \% \text { CC; } 29 \% \text { rupture; } 6 \% \\
\text { infection }\end{array}$ & $\begin{array}{c}62 \% \text { fTRAM; } 21 \% \\
\text { SGAP; } 17 \% \text { LD }\end{array}$ & $1(1.9)$ & $3(5.8)$ & $6(11.6)$ & - \\
\hline
\end{tabular}

${ }^{*}$ Complication rate reported as a percentage of the total number of flaps; ${ }^{*}$ includes breast related operations performed to improve aesthetic outcome or symmetry, not including nipple reconstruction; ${ }^{c}$ reported revisions required in patients with unilateral reconstructions only $(n$ = 16). CC: capsular contracture; DIEP: deep inferior epigastric perforator flap; fTRAM: free transverse rectus abdominis myocutaneous flap; msTRAM, muscle sparing TRAM; SIEA: superficial inferior epigastric artery flap; IGAP: inferior gluteal artery perforator flap; SGAP: superior gluteal artery perforator flap; TMG: transverse myocutaneousgracilis flap; LD: latissimus dorsi flap; TUG: transverse upper gracilis flap; DPM: dual pedicle dermoparenchymalmastopexy

BMI was $24.7 \mathrm{~kg} / \mathrm{m}^{2}$. Prior to autologous salvage, 23 $(42.6 \%)$ patients received neoadjuvant or adjuvant radiotherapy. Initial autologous reconstructions consisted of 18 (33.3\%) pedicle TRAM flaps, 14 (25.9\%) DIEP flaps, 12 LD flaps $(22.2 \%), 4(7.4 \%)$ free TRAM flaps, 3 (5.6\%) SGAP flaps, 2 (3.7\%) SIEA flaps, and $1(1.9 \%)$ TMG flap. The most common indications for autologous salvage included total flap loss in $30(55.6 \%)$ breasts, partial flap loss in $16(29.6 \%)$ breasts, and fat necrosis in $4(7.4 \%)$ breasts. The most common flap utilized for tertiary reconstruction was the LD flap which was used in 31 breasts $(57.4 \%)$. Other flaps reported include the contralateral free LD flap in $12(22.2 \%)$ breasts, SGAP in $5(9.3 \%)$ breasts, TMG in $3(5.6 \%)$ breasts, thoracodorsal artery perforator flap (TDAP) in $2(3.7 \%)$ breasts, and DIEP flap in $1(1.9 \%)$ breast.
Four studies included outcomes following tertiary reconstruction using pedicle flaps (33 patients) ${ }^{[8,20,28,29]}$ and 2 studies included microsurgical free flaps (21 patients $)^{[8,16]}$. Of the studies utilizing free flaps, total flap loss was reported in 2 breasts (9.5\%; range $0-22.2 \%$ ), one occurring with a SGAP flap and the other with a TMG flap. One partial flap loss $(4.8 \%$; range $0-8.3 \%)$ occurred following reconstruction with a contralateral free LD flap. No total or partial flap loss was reported with pedicle LD flap reconstruction. Revisional surgery to improve aesthetic outcome occurred in 23 breasts (70.0\%; range $42.8-92.9 \%)$. Total complications were reported in 19 breasts (42.2\%; range 14.2-64.3\%). Complications besides total/partial flap failure included one hematoma $(2.1 \%$; range $0-7.1 \%)$, one infection (2.1\%; range $0-8.3 \%)$, 11 seroma $(23.4 \%$; range 7.1 $57.1 \%)$, and 2 wound healing problems $(6.5 \%$; range 
Table 3: Studies reporting surgical outcomes following restoration of previously failed or unsatisfactory autologous reconstruction

\begin{tabular}{|c|c|c|c|c|c|c|c|c|}
\hline \multirow[b]{2}{*}{ Authors } & \multirow[b]{2}{*}{$\begin{array}{l}\text { Breasts } \\
\text { (n) }\end{array}$} & \multirow[b]{2}{*}{$\begin{array}{c}\text { Initial } \\
\text { reconstruction }\end{array}$} & \multirow[b]{2}{*}{$\begin{array}{l}\text { Indication for } \\
\text { salvage }\end{array}$} & \multirow[b]{2}{*}{ Flap choice } & \multicolumn{4}{|c|}{ Surgical outcomes } \\
\hline & & & & & $\begin{array}{c}\text { Anastomotic } \\
\text { revisions, } \\
n(\%)\end{array}$ & $\begin{array}{c}\text { Total } \\
\text { flap } \\
\text { loss, } \\
n(\%)\end{array}$ & $\begin{array}{c}\text { Total } \\
\text { complications } \\
n(\%)\end{array}$ & $\begin{array}{c}\text { Revisional } \\
\text { surgery } \\
n(\%)\end{array}$ \\
\hline Munhoz et al. ${ }^{[16]} 2016$ & 12 & Pedicle LD & Total flap loss & $\begin{array}{c}\text { Contralateral } \\
\text { free LD }\end{array}$ & 0 & 0 & $5(41.7)$ & $7(58.3)$ \\
\hline Mohan et al. ${ }^{[20]} 2013$ & 7 & $\begin{array}{l}\text { 86\% DIEP; } 14 \% \\
\text { TMG }\end{array}$ & Total flap loss & $\begin{array}{l}\text { LD +/- } \\
\text { implant }\end{array}$ & - & 0 & - & - \\
\hline Hamdi et al. ${ }^{[8]} 2010$ & 14 & $\begin{array}{l}\text { 57\% DIEP; } 21 \% \\
\text { SGAP; } 14 \% \text { SIEA; } \\
7 \% \text { msTRAM }\end{array}$ & $\begin{array}{l}79 \% \text { total } \\
\text { flap loss; } \\
14 \% \text { partial } \\
\text { flap loss; } 7 \% \\
\text { recurrence }\end{array}$ & $\begin{array}{l}\text { 36\% SGAP; } \\
\text { 21\% TMG; } \\
21 \% \text { LD; } \\
\text { 14\% TDAP; } \\
9 \% \text { DIEP }\end{array}$ & - & $2(14.2)$ & $4(28.6)$ & $13(92.9)$ \\
\hline Hammond et al. ${ }^{[28]} 2007$ & 14 & pTRAM & Partial flap loss & LD & - & 0 & $9(64.2)$ & - \\
\hline Karanas et al. ${ }^{[29]} 2002$ & 7 & $\begin{array}{c}56 \% \text { fTRAM; } 44 \% \\
\text { pTRAM }\end{array}$ & $\begin{array}{c}57 \% \text { fat } \\
\text { necrosis; } 14 \% \\
\text { radiation; } 14 \% \\
\text { insufficient } \\
\text { volume; } 14 \% \\
\text { chest wall } \\
\text { depression }\end{array}$ & LD & - & 0 & $1(14.3)$ & $3(42.9)$ \\
\hline
\end{tabular}

*Complication rate reported as a percentage of the total number of flaps; "includes breast related operations performed to improve aesthetic outcome or symmetry, not including nipple reconstruction. LD: latissimus dorsi flap; DIEP: deep inferior epigastric perforator flap; TMG: transverse myocutaneousgracilis flap; SGAP: superior gluteal artery perforator flap; SIEA: superficial inferior epigastric artery flap; msTRAM: muscle sparing transverse rectus abdominis myocutaneous flap; pTRAM: pedicle transverse rectus abdominis myocutaneous flap; fTRAM: free transverse rectus abdominis myocutaneous flap; TDAP: thoracodorsal artery perforator flap

0-7.1\%). No instances of microsurgical revisions or fat necrosis were reported.

Two studies evaluated patient satisfaction (17 patients) and no studies directly evaluated aesthetic outcomes in patients with restoration of previously failed autologous reconstruction ${ }^{[28,29]}$. Of these, one study determined patient satisfaction following LD salvage of partial TRAM loss via telephone interview and stated that all 10 patients interviewed "found the procedure worthwhile" ${ }^{\text {[28] }}$. The other study also evaluated satisfaction after LD salvage of partial TRAM loss and reported a $100 \%$ patient satisfaction rate $(n=7)^{[29]}$.

\section{DISCUSSION}

Failure of post-mastectomy breast reconstruction can be a devastating experience for patients ${ }^{[15]}$. Evaluating outcomes of techniques to salvage reconstruction is crucial to optimizing their management and enhancing quality of life. Tertiary reconstruction via use of autogenous tissue has been suggested to provide improved outcomes in these patients by several studies $^{[6-8]}$. However, as demonstrated in this review, much of the data pertaining to tertiary reconstruction is limited to case series that suffer from their retrospective nature, limitations in sample size, and institutional variability. The purpose of this systematic review was to consolidate surgical outcomes, aesthetic outcomes, and patient satisfaction of tertiary reconstruction in order to better characterize its benefits and pitfalls.

Tertiary reconstruction is best described following failed or unsatisfactory prosthetic breast reconstruction. In this review, $79 \%(15 / 19)$ of included studies evaluated outcomes of tertiary reconstruction in this setting. Since 2002, prosthetic reconstruction has surpassed autologous tissue as the leading reconstructive modality and its use has continued to grow in recent years ${ }^{[12]}$. The reason for this is multifactorial but likely related to its technical feasibility, lack of donor site morbidity, and changes in mastectomy patterns, such as increased bilateral mastectomies. Despite these advantages, risk of potential complications and unsatisfactory long term aesthetic outcomes are significantly increased as compared to autologous reconstruction ${ }^{[13,30]}$. In this review, capsular contracture, poor cosmesis, persistent pain, and infection were the most commonly cited reasons for patients choosing to undergo tertiary reconstruction. History of neoadjuvant or adjuvant radiotherapy has been shown to significantly increase the risk of these adverse outcomes and was present in nearly half of patients who underwent autologous conversion in this review.

Prior implant placement and resulting capsule formation 
may increase the complexity of autologous breast reconstruction. It has previously been reported that recipient vessel scarring was 5.23 times more likely in patients with prior prosthestic reconstruction at the time of autologous conversion as compared to those with de novo autologous reconstruction ${ }^{[10]}$. However, the impact of prior implant reconstruction on outcomes of autologous conversion remains unclear. Planned autologous conversion after immediate tissue expander reconstruction and adjuvant radiotherapy, termed delayed-immediate reconstruction, has been described by several studies as a method of preserving the tissue envelope while avoiding deleterious effects of radiation on the flap ${ }^{[31-33]}$. In a series of 16 delayedimmediate reconstructions, Kronowitz et al. ${ }^{[31]}$ reported intra-arterial thrombosis in $12.5 \%$ of breasts following autologous conversion, however, no patients suffered a flap loss. In a larger study, Patel et al. ${ }^{[33]}$ compared outcomes of autologous conversion as a part of the delayed-immediate protocol in 74 reconstructions to 118 delayed autologous reconstructions after radiation with no prior prosthesis insertion. They reported no difference in anastomotic revisions $(6.8 \%$ vs. $5.9 \% ; P$ $=1.0)$ or rate of flap failure $(4.1 \%$ vs. $2.5 \% ; P=0.68)$. The literature pertaining to unplanned autologous conversion secondary to implant-related complications has suggested similar success but is limited. Only one study included in this review provided a headto-head comparison between autologous conversion after unsuccessful prosthetic reconstruction to patients with de novo autologous reconstruction ${ }^{[10]}$. Roostaeian et al. ${ }^{[10]}$ reported no difference in rates of flap loss or operative take back between the two groups, but did note an increase in major complications in the group with a prior prosthesis $(17.4 \%$ vs. $8.1 \%$; $P$ $=0.035$ ). Consistent with these studies, pooled-analysis in this review demonstrated a low incidence of total flap loss or microsurgical revisions in $1.6 \%$ and $3.2 \%$, respectively. Overall, there is little evidence to suggest that prior prosthetic reconstruction negatively impacts later autologous reconstruction in the setting of tertiary reconstruction or the delayed-immediate protocol. However, future prospective studies are required with larger sample sizes to fully understand the impact of unsuccessful prosthetic reconstruction on autologous conversion.

In addition to salvaging breast reconstruction, autologous conversion in the setting of tertiary reconstruction may provide an improved long-term aesthetic result and satisfaction ${ }^{[30]}$. In this review, 2 studies compared aesthetic outcomes of patients with autologous conversion to patients that completed prosthetic reconstruction ${ }^{[19,25]}$. Mioton et al. ${ }^{[19]}$ reported superior scores across 4 out of 5 domains evaluated (volume, contour, placement, and inframammary fold) in 18 patients with autologous conversion after complicated implant reconstruction as compared to 136 patients with uncomplicated prosthetic reconstruction. Spear and Onyewu ${ }^{[25]}$ compared aesthetic scores of patients with autologous conversion after irradiated prosthetic reconstruction to patients with non-irradiated prosthetic reconstruction and reported similar aesthetic means of 3.25 and 3.28 (4-point scale), respectively. In this review, all 5 included studies demonstrated acceptable rates of positive aesthetic results and patient satisfaction following tertiary reconstruction in the setting of unsuccessful prosthetic reconstruction ${ }^{[11,19,20,25,27]}$. This suggests that improved aesthetic result associated with autologous breast reconstruction may be attained even in patients with prior unsuccessful prosthetic reconstruction.

Studies reporting outcomes following restoration of a previously failed autologous breast reconstruction utilizing a second flap are limited and make it difficult to draw any definitive conclusions. In this review, all 5 studies that met inclusion criteria retrospectively assessed outcomes of less than 15 patients $^{[8,16,20,28,29]}$. Of these, 4 studies reported outcomes of unsuccessful autologous reconstruction by utilizing a pedicle LD flap ${ }^{[8,20,28,29]}$. Overall, no partial or total flap loss was observed in these patients. Only 2 included studies evaluated the utility of microsurgical free flap reconstruction in the setting of previous total flap loss. Hamdi et al. ${ }^{[8]}$ assessed outcomes following second free flap reconstruction after prior flap loss and noted total flap loss in 2 of 9 patients (22.5\%). In another series, Munhoz et al. ${ }^{[16]}$ evaluated outcomes following the use of a contralateral free LD flap to salvage breast reconstruction after previous LD breast reconstruction failure. They reported 1 partial flap loss $(8.3 \%)$ but no total flap loss. Overall, the available data suggests that a second free flap after prior free flap failure is a feasible technique to salvage breast reconstruction in select patients. However, future studies with significantly increased sample sizes are required in order to better define outcomes and determine the optimal approach to managing patients with unsuccessful autologous breast reconstruction. In addition, data evaluating aesthetic outcomes, patient satisfaction, or quality of life are lacking and is necessary to evaluate the potential benefits that tertiary reconstruction may provide.

Tertiary reconstruction in the setting of prior failed autologous breast reconstruction brings several challenges that need to be addressed to ensure its success. The previous failure must be analyzed in order to adjust strategy and eliminate potential 
causes of failure for the new flap. Previously cited considerations for attempting microsurgical free flap reconstruction in the setting of prior free flap failure include reassessment of the preoperative preparations, flap choice, the recipient vessels and anastomotic technique, the patient's coagulability and potential for thrombosis, the appropriateness of intraoperative positioning, the postoperative care, and the surgeon's own level of experience ${ }^{[34]}$. Full blood and coagulation tests with consultation of hematologist may also be required, particularly in those patients without evidence of obvious technical problem as the cause of flap failure. Hamdi et al ${ }^{[8]}$ reported underlying hematologic disorders in 3 out of 14 patients who underwent tertiary breast reconstruction after failed free flap reconstruction. Two of these patients went on to have successful breast restoration with the use of a second free flap and the other patient underwent successful implant reconstruction. This suggests that with proper medical management, successful microsurgical restoration in these patients may be attained.

This is the first systematic review to evaluate the literature and consolidate the available data concerning autogenous tissue transfer to salvage unsuccessful breast reconstruction. However, there are several inherent limitations in this systematic review, including retrospective study design and the biases within each of the studies included. Inconsistently reported data and scarce reporting of patient comorbidities also limited the findings of this review. The majority of current studies report outcomes from a single institution, many of which significantly differ in their approach of managing patients with unsuccessful breast reconstruction. Outcomes are often heterogeneously reported precluding a true meta-analysis. A benefit of this manuscript is that it gives a general perspective of the surgical success and aesthetic result that may be obtained with tertiary breast reconstruction. The authors acknowledge that there are many factors that contribute to a patient's decision when assessing whether to reattempt breast reconstruction after experiencing an adverse outcome with a past attempt. Based on this data, autologous conversion in the setting of unsuccessful prosthetic breast reconstruction appears to be valuable option to improving outcomes in these patients. In addition, data describing autologous breast reconstruction in the setting of a previous unsuccessful attempt is extremely limited but suggests it is a viable method to salvage breast reconstruction in appropriate patients. This systematic review identifies the risk of complications and reconstructive failure associated with tertiary reconstruction, stressing the importance of proper patient selection when contemplating breast reconstruction in the setting of past unsuccessful breast reconstruction.

\section{DECLARATIONS}

\section{Authors' contributions}

Conception and design of review, performed literature search protocol, data collection, drafting of manuscript, final revisions, and final approval of manuscript: B. ELSabawi

Design of search protocol, study selection, review of data, data analysis and interpretation, drafting of manuscript, final revisions and final approval of manuscript: A.C. Howell

Design of review, study selection, data interpretation, drafting of manuscript, final revisions, and final approval of manuscript: K.M. Patel

\section{Financial support and sponsorship None.}

\section{Conflicts of interest}

There are no conflicts of interest.

\section{Patient consent}

Not applicable.

\section{Ethics approval}

Not applicable.

\section{REFERENCES}

1. Wilkins EG, Cederna PS, Lowery JC, Davis JA, Kim HM, Roth RS, Goldfarb S, Izenberg PH, Houin HP, Shaheen KW. Prospective analysis of psychosocial outcomes in breast reconstruction: oneyear postoperative results from the Michigan Breast Reconstruction Outcome Study. Plast Reconstr Surg 2000;106:1014-25.

2. Atisha D, Alderman AK, Lowery JC, Kuhn LE, Davis J, Wilkins EG Prospective analysis of long-term psychosocial outcomes in breast reconstruction: two-year postoperative results from the Michigan Breast Reconstruction Outcomes Study. Ann Surg 2008;247:1019-28.

3. Chao LF, Patel KM, Chen SC, Lam HB, Lin CY, Liu HE, Cheng $\mathrm{MH}$. Monitoring patient-centered outcomes through the progression of breast reconstruction: a multicentered prospective longitudinal evaluation. Breast Cancer Res Treat 2014;146:299-308.

4. Brandberg Y, Malm M, Blomqvist L. A prospective and randomized study "SVEA" comparing effects of three methods for delayed breast reconstruction on quality of life, patient-defined problem areas of life, and cosmetic result. Plast Reconstr Surg 2000;105:66-74.

5. Massenburg BB, Sanati-mehrizy P, Ingargiola MJ, Rosa JH, Taub PJ. Flap failure and wound complications in autologous breast reconstruction: a national perspective. Aesthetic Plast Surg 2015;39:902-9.

6. Spear SL, Masden D, Rao SS, Nahabedian MY. Long-term outcomes of failed prosthetic breast reconstruction. Ann Plast Surg 2013;71:28691.

7. Hamdi M, Casaer B, Andrades P, Thiessen F, Dancey A, D’Arpa S, Van Landuyt K. Salvage (tertiary) breast reconstruction after implant failure. J Plast Reconstr Aesthet Surg 2011;64:353-9.

8. Hamdi M, Andrades P, Thiessen F, Stillaert F, Roche N, Van Landuyt $\mathrm{K}$, Monstrey S. Is a second free flap still an option in a failed free flap 
breast reconstruction? Plast Reconstr Surg 2010;126:375-84.

9. Levine SM, Lester ME, Fontenot B, Allen RJ. Perforator flap breast reconstruction after unsatisfactory implant reconstruction. Ann Plast Surg 2011;66:513-7.

10. Roostaeian J, Yoon AP, Ordon S, Gold C, Crisera C, Festekjian J, Da Lio A, Lipa JE. Impact of prior tissue expander/implant on postmastectomy free flap breast reconstruction. Plast Reconstr Surg 2016;137:1083-91.

11. Visser NJ, Damen TH, Timman R, Hofer SO, Mureau MA. Surgical results, aesthetic outcome, and patient satisfaction after microsurgical autologous breast reconstruction following failed implant reconstruction. Plast Reconstr Surg 2010;126:26-36.

12. Albornoz CR, Bach PB, Mehrara BJ, Disa JJ, Pusic AL, McCarthy CM, Cordeiro PG, Matros E. A paradigm shift in U.S. breast reconstruction: increasing implant rates. Plast Reconstr Surg 2013;131:15-23.

13. El-Sabawi B, Sosin M, Carey JN, Nahabedian MY, Patel KM. Breast reconstruction and adjuvant therapy: a systematic review of surgical outcomes. J Surg Oncol 2015;112:458-64

14. Cordeiro PG, Albornoz CR, Mccormick B, Hu Q, Van zee K. The impact of postmastectomy radiotherapy on two-stage implant breast reconstruction: an analysis of long-term surgical outcomes, aesthetic results, and satisfaction over 13 years. Plast Reconstr Surg 2014;134:588-95.

15. Higgins KS, Gillis J, Williams JG, Leblanc M, Bezuhly M, Chorney JM. Women's experiences with flap failure after autologous breast reconstruction: a qualitative analysis. Ann Plast Surg 2017;78:521-5.

16. Munhoz AM, Montag E, Arruda EG, Okada A, Fonseca A, Filassi JR, Gemperli R. The use of contralateral free extended latissimus dorsi myocutaneous flap for a tertiary failed breast reconstruction: is it still an option? J Plast Reconstr Aesthet Surg 2016;69:1087-91.

17. Moher D, Liberati A, Tetzlaff J, Altman DG; PRISMA Group. Preferred reporting items for systematic reviews and meta-analyses: the PRISMA statement. Int J Surg 2010;8:336-41.

18. Pülzl P, Huemer GM, Schoeller T. Transverse musculocutaneous gracilis flap for treatment of capsular contracture in tertiary breast reconstruction. Ann Plast Surg 2015;74:167-72.

19. Mioton LM, Seth A, Gaido J, Fine NA, Kim JY. Tracking the aesthetic outcomes of prosthetic breast reconstructions that have complications. Plast Surg (Oakv) 2014;22:70-4

20. Mohan AT, Al-Ajam Y, Mosahebi A. Trends in tertiary breast reconstruction: literature review and single centre experience. Breast 2013;22:173-8.

21. Rabey NG, Lie KH, Kumiponjera D, Erel E, Simcock JW, Malata CM. Salvage of failed prosthetic breast reconstructions by autologous conversion with free tissue transfers. Eplasty 2013;13:e32.

22. Peled AW, Stover AC, Foster RD, Mcgrath MH, Hwang ES. Long-term reconstructive outcomes after expander-implant breast reconstruction with serious infectious or wound-healing complications. Ann Plast Surg 2012;68:369-73

23. Gurunluoglu R, Shafighi M, Schwabegger A, Ninkovic M. Secondary breast reconstruction with deepithelialized free flaps from the lower abdomen for intractable capsular contracture and maintenance of breast volume. J Reconstr Microsurg 2005;21:35-41.

24. Mosahebi A, Atherton D, Ramakrishnan V. Immediate bilatera autologous breast reconstruction for silicone intolerance. $\mathrm{Br} J$ Plast Surg 2005;58:714-6.

25. Spear SL, Onyewu C. Staged breast reconstruction with salinefilled implants in the irradiated breast: recent trends and therapeutic implications. Plast Reconstr Surg 2000;105:930-42.

26. Weiss PR, Ship AG. Restoration of breast contour with autologous tissue after removal of implants. Ann Plast Surg 1995;34:236-41.

27. Feng LJ, Mauceri K, Berger BE. Autogenous tissue breast reconstruction in the silicone-intolerant patient. Cancer 1994;74:440-9.

28. Hammond DC, Simon AM, Khuthaila DK, Hoberman L, Sohn S Latissimus dorsi flap salvage of the partially failed TRAM flap breast reconstruction. Plast Reconstr Surg 2007;120:382-9.

29. Karanas YL, Santoro TD, Shaw WW, Da Lio AL. Use of the latissimus dorsi flap for recontouring and augmentation after TRAM flap breast reconstruction. Ann Plast Surg 2002;48:343-7.

30. Hu ES, Pusic AL, Waljee JF, Kuhn L, Hawley ST, Wilkins E, Alderman AK. Patient-reported aesthetic satisfaction with breast reconstruction during the long-term survivorship period. Plast Reconstr Surg 2009;124:1-8

31. Kronowitz SJ, Hunt KK, Kuerer HM, Babiera G, McNeese MD Buchholz TA, Strom EA, Robb GL. Delayed-immediate breast reconstruction. Plast Reconstr Surg 2004;113:1617-28.

32. Kronowitz SJ, Lam C, Terefe W, Hunt KK, Kuerer HM, Valero V, Lance S, Robb GL, Feng L, Buchholz TA. A multidisciplinary protocol for planned skin-preserving delayed breast reconstruction for patients with locally advanced breast cancer requiring postmastectomy radiation therapy: 3-year follow-up. Plast Reconstr Surg 2011;127:2154-66.

33. Patel KM, Albino F, Fan KL, Liao E, Nahabedian MY. Microvascular autologous breast reconstruction in the context of radiation therapy: comparing two reconstructive algorithms. Plast Reconstr Surg 2013;132:251-7.

34. Baumeister S, Follmar KE, Zenn MR, Erdmann D, Levin LS. Strategy for reoperative free flaps after failure of a first flap. Plast Reconstr Surg 2008;122:962-71. 